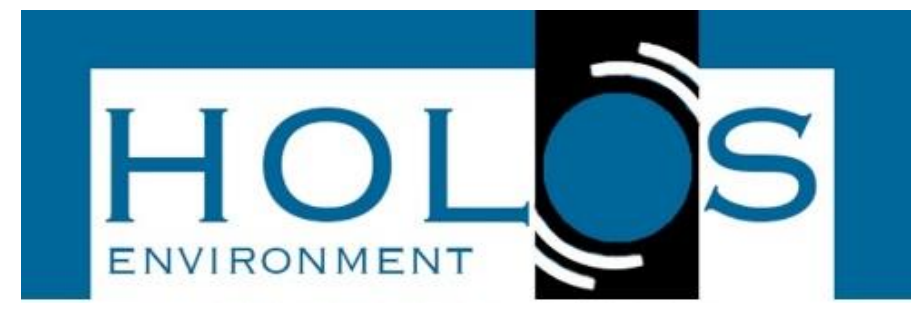

\title{
ESTUDO DA APLICACÃ̃ DE COBERTURAS VERDES NO OBJETIVO DE SE CONSTRUIR EDIFÍCIOS NEUTROS EM CARBONO
}

\section{STUDY OF GREEN ROOFS APLICATIONS IN ORDER TO DELIVER BUILDINGS NEUTRAL CARBON}

\author{
José de Almendra Freitas Jr. ${ }^{1}$, Carlos Roberto Sanquetta ${ }^{1}$, Setsuo Iwakiri ${ }^{1}$, Marienne \\ Do Rocio de Mello Maron da Costa ${ }^{1}$, Henrique Soares Koehler ${ }^{1}$
}

Artigo recebido em: 13/06/2016 e aceito para publicação em: 22/03/2017.

DOI: http://dx.doi.org/10.14295/holos.v17i1.11494

Resumo: No contexto das mudanças climáticas o setor da construção civil vem se envolvendo em programas de mitigação e neutralização de emissões de gases do efeito estufa (GEE). Uma das formas possíveis de "neutralizar" parte das emissões é transformar as edificações em reservatórios de carbono por meio da implantação de "coberturas verdes", que consiste no cultivo de vegetação em substratos de terra na cobertura dos edifícios. Ocorre que esta implantação acrescenta carga à estrutura da edificação, havendo a necessidade desta ser reforçada. Este trabalho, por meio de um estudo de caso representativo, ajuda a analisar se o acréscimo de emissões de $\mathrm{CO}_{2}$ decorrente do reforço estrutural é menor que o carbono armazenado na "cobertura verde", verificando a viabilidade desta técnica neste aspecto. $\mathrm{O}$ trabalho demonstrou que para o estudo de caso, esta implantação tem saldo muito positivo quanto às emissões de $\mathrm{CO}_{2}$, armazenando muito mais carbono que emitindo, podendo neutralizar de forma significativa as emissões decorrentes da construção da obra.

Palavras-chave: Cobertura verde. Emissão de $\mathrm{CO}_{2}$. Construção.

Abstract: In the context of climate change, the industrial sectors have been involved in mitigation and neutralization programs of emissions of greenhouse gases (GHGs). One way with the construction industry has to "neutralize" some of their emissions is to transform the building in carbon sinks through the implementation of "green roofs, "which consists of the vegetation growing on land substrates on the roof of buildings. It happens that this deployment adds load to the building structure, with the need for this to be strengthened. This work, through a study of representative case, helps to examine whether the addition of $\mathrm{CO}_{2}$ emission resulting from the structural reinforcement is less than the carbon stored in the "green roofs", verifying the feasibility of the this technique in this regard. The study showed that for the case study, this deployment has very positive balance as $\mathrm{CO}_{2}$ emissions by storing more carbon than emitting and can significantly neutralize the emissions from the construction work.

Keywords: Green roof. $\mathrm{CO}_{2}$ emissions. Construction.

\footnotetext{
${ }^{1}$ Universidade Federal do Paraná (UFPR). Emails: (freitasjose@terra.com.br, carlos sanquetta@hotmail.com, setsuo.ufpr@gmail.com, marienne@ufpr.br, koehler@ufpr.br)
} 


\section{INTRODUÇÃO}

Devido aos crescentes desafios ambientais que a sociedade atual vem enfrentando, o problema do efeito estufa e o aumento das concentrações do $\mathrm{CO}_{2}$ na atmosfera vem se tornando cada vez mais relevante e preocupando as principais nações do mundo. Dentro deste contexto muitos países desenvolvidos (ou cidades de forma individual) tem implementado regulamentos especificamente destinados a controlar as emissões de carbono pelos edifícios, tanto na fase de uso como para a fase de construção. O desafio atual para a indústria da construção civil destas nações é alcançar o objetivo de produzir edifícios "neutros em carbono" a partir de 2020, o que significa alcançar o equilíbrio entre as quantidades de carbono emitidas e seqüestradas ou armazenadas. A definição "neutralização de carbono" é usada internacionalmente no sentido da redução e/ou compensação do carbono emitido por uma atividade. O Brasil, embora ainda não tenha atingido este nível de ações, já têm construtoras e incorporadoras que vem se preocupando com este problema e voluntariamente tomando ações e buscando caminhos para minimizar as suas emissões.

Em 2009 a ONU, por meio da UNEP (United Nations Environment Programme) colocou para discussões na 15 a Conferência das Partes (COP 15), como assunto relevante, que as construções são fonte de importantes emissões e que é o setor industrial que tem o maior potencial para alcançar reduções significativas e de baixo custo de emissões de GEE. A UNEP salientava que os países não conseguirão cumprir as metas de redução de emissões sem aumentos de eficiência energética na construção e que este setor industrial já está empenhado em muitos países em desempenhar um papel de liderança nas reduções de emissões (UNEP, 2009). Os levantamentos de emissões dos GEE relativos à produção das edificações, como para qualquer produto industrial, devem seguir as normas NBR ISO 14025:2015, NBR ISO 14064:2007, NBR ISO 14040:2009 versão corrigida 2014 e NBR ISO 14044:2009 Errata 1:2014. Estas normas recomendam também que se relate 0 carbono armazenado, quando este é de origem biogênica, não fóssil.

O uso de materiais de construção de madeira assim como a fixação de carbono de origem não fóssil dentro de uma edificação tem grande potencial para contrabalançar ou neutralizar as inevitáveis emissões decorrentes do processo 
construtivo. Isto se deve ao fato que de 40 a $45 \%$ da massa da madeira é composta pelo elemento químico carbono $(\mathrm{C})$ e como este representa apenas uma fração da molécula de $\mathrm{CO}_{2}$, portanto cada unidade de massa de carbono fixada representa a não emissão de 3,667 unidades de massa de $\mathrm{CO}_{2}$ (OLIVEIRA et al., 2011).

Este trabalho mostra, por meio de um estudo de caso, que três abordagens diferentes de "coberturas verdes" podem compensar boa parte das emissões de GEE que ocorrem durante a fase de construção de um edifício, e prova também, que o reforço da estrutura do edifício necessário para suporta-las causa bem menos emissões que neutraliza. Estas coberturas, além de capturarem e armazenarem carbono em sua vegetação, como é obviamente intuitivo, servem também como reservatório de carbono no seu solo de substrato, contrabalançando parte dos efeitos da remoção do solo original do terreno da obra.

Rowe (2010) define uma "cobertura verde" como o cultivo de vegetação em solo vegetal em cima de um edifício, salientando que estas proporcionam benefícios ambientais e reduzem as nas emissões de $\mathrm{CO}_{2}$ pelas construções, capturando e fixando o carbono na vegetação e armazenando carbono no substrato, além de melhorar o desempenho térmico das edificações. Santamouris (2014) coloca que as "coberturas verdes" minimizam os efeitos das mudanças climáticas nas cidades, reduzindo aumentos de temperatura decorrentes da formação de ilhas de calor. Li e Yeung (2014) reportam que estas melhoram o desempenho dos equipamentos de ar condicionado, minimizando também os picos de descarga de águas pluviais. Garrison e Horowitz (2012) colocam que as "coberturas verdes" podem desempenhar um pequeno papel na redução $\mathrm{CO}_{2}$ na a atmosfera, devido ao carbono que é armazenado no solo ou substrato, assim como do carbono sequestrado pela vegetação.

Devido ao fato de que a implantação de uma "cobertura verde" acrescenta carga na estrutura da edificação, esta precisa ser reforçada para se suportar este peso extra. Este estudo de caso analisa especificamente os acréscimos de $\mathrm{CO}_{2}$ decorrentes deste reforço, comparando-se com o carbono armazenado na "cobertura verde".

No Brasil ainda não é usual a adoção de "coberturas verdes" nos edifícios, devido a características culturais, de custos, da necessidade de manutenção entre outras, mas que devido a crescente necessidade de mitigar as 
emissões dos GEE seria interessante mudar esta prática. Salienta-se que há uma grande carência de estudos que proporcionem um melhor dimensionamento dos benefícios das "coberturas verdes" quanto a mitigação e neutralização das emissões de GEE.

Este trabalho visa demonstrar que a implantação de uma "cobertura verde" sobre um edifício residencial pode neutralizar de forma significativa as emissões de $\mathrm{CO}_{2}$ decorrentes da construção da obra.

\section{MATERIAL E MÉTODOS}

São muitas as formas arquitetônicas com as quais se pode colocar uma "cobertura verde" em um edifício. Para se alcançar o objetivo deste trabalho, optou-se por analisar um estudo de caso representativo. Para tanto foi selecionado um projeto de um edifício residencial tradicional, com quatro pavimentos com estrutura em concreto armado. O projeto do edifício contempla a possibilidade de se instalar dois terraços na cobertura nos quais são passíveis se instalar "coberturas verdes" que totalizam uma área de $352,38 \mathrm{~m}^{2}$. O esquema da planta de cobertura está mostrado na Figura 1.

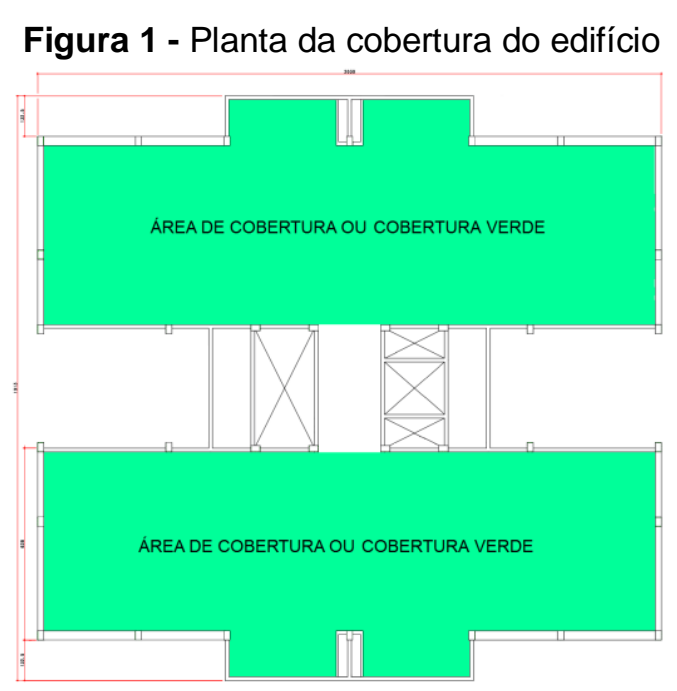

Dentro do estudo de caso, foi projetada a estrutura para o edifício de quatro formas, uma com cobertura de laje impermeabilizada e três com variações de "coberturas verdes", de diferentes substratos e vegetações, para todos, simulando análises de ciclo de vida (ACV), foram levantadas as quantidades de emissões de $\mathrm{CO}_{2}$. 
Para as três alternativas de "coberturas verdes", este estudo determinou a quantidade de carbono armazenado nos solos utilizados como substratos para este e na vegetação.

Com os totais de carbono armazenado e as emissões de $\mathrm{CO}_{2}$ da implantação das variações da estrutura, procederam-se balanços e análises comparativas.

\subsection{Definições das alternativas de "coberturas verdes"}

Analisaram-se três tipos diferentes de "coberturas verdes" dentro da classificação citada em Wark e Wark (2003), que são: extensivas, semi-intensivas e intensivas, cujas especificações básicas são:

- Extensiva, tipo mais simples, apropriada para ervas e gramíneas, exige uma espessura de solo do sistema de 6 a $20 \mathrm{~cm}$, necessitando de uma baixa manutenção e não tem irrigação;

- Semi-intensiva, apropriado para gramíneas e arbustos, exige uma espessura de solo do sistema de 12 a $25 \mathrm{~cm}$, necessitando de manutenção periódica;

- Intensiva, suporta arbustos e árvores exigindo uma espessura de solo do sistema de 15 a $40 \mathrm{~cm}$, exigindo uma manutenção alta, com irrigação regular.

As variações da estrutura do edifício foram dimensionadas para cada alternativa de forma a suportar a carga decorrente do somatório dos pesos da impermeabilização, drenagem, do substrato de solo, da vegetação e da manutenção. Cada uma gera um carregamento que corresponde com o tipo e uso da "cobertura verde" desejada. Para se projetar a estrutura de um edifício, foram consideradas as cargas permanentes e as cargas acidentais, conforme a NBR 6120:1980.

As cargas permanentes incluem o peso-próprio da estrutura, acrescidas do peso do conjunto de materiais que compõe as camadas do sistema da "cobertura verde" sobre uma laje. As camadas são: o sistema de impermeabilização com sua proteção, a drenagem, o substrato ou a camada de terra ou solo dão suporte à vegetação e a cobertura vegetal propriamente dita. Às cargas permanentes deve ser acrescido ainda o peso da água retida. 
A NBR 6120:1980 considera para dimensionamento estrutural de edifícios, a carga para terra compactada, de $1.800 \mathrm{kgf} / \mathrm{m}^{3}$. Para o caso de uma "cobertura verde", pode-se considerar $1.550 \mathrm{kgf} / \mathrm{m}^{3}$, que seria a carga para terra vegetal úmida com $15 \%$ de umidade. Para se estimar as cargas permanentes dos três tipos de "coberturas verdes" foi considerada a carga de $1.550 \mathrm{kgf} / \mathrm{m}^{3}$ para o substrato de solo e estimando a espessura média do sistema de impermeabilização em $12 \mathrm{~cm}$ e uma carga permanente para esta camada em $250 \mathrm{kgf} / \mathrm{m}^{2}$. Cabe ressaltar que esta carga é a mesma que seria utilizada para representar uma cobertura em telhas de fibrocimento comum sobre uma laje de concreto armado.

Baseando-se nas colocações de Johnson e Newton (1991), e nos tipos de coberturas, este estudo utilizou as espessuras dos substratos em $15 \mathrm{~cm}$ para 0 extensivo, $25 \mathrm{~cm}$ para o semi-intensivo e $40 \mathrm{~cm}$ para o intensivo. Cabe salientar, que substratos de maior espessura, não somente suportam uma vegetação de maior porte, como também tem melhor desempenho como isolante térmico e acústico (CASTLETON et al., 2010).

O valor da carga acidental, como está definido na NBR 6.120:1980 se refere ao carregamento decorrente da circulação de pessoas e equipamentos que farão ao uso da cobertura, que recomenda considerar a carga acidental de $300 \mathrm{~kg} / \mathrm{m}^{2}$ para áreas públicas com acesso livre de pessoas, em áreas com acesso somente para manutenção, recomenda a carga de $50 \mathrm{kgf} / \mathrm{m}^{2}$.

Com o intuito de se proceder ao comparativo de impacto dos acréscimos carbono foram calculadas as emissões de $\mathrm{CO}_{2}$ decorrentes da construção das quatro alternativas da estrutura do edifício, que foram identificados por CL para cobertura somente com laje impermeabilizada, CV1 com "cobertura verde" extensiva $(15 \mathrm{~cm}$ de espessura), CV2 com semi-intensiva (25 cm de espessura) e CV3 com intensiva (40 $\mathrm{cm}$ de espessura). Em todas as alternativas de carga o estudo considerou a cobertura sem acesso aberto ao público, somente para pessoal de manutenção.

Para se desenvolver o comparativo do impacto de carbono, se dimensionou a estrutura do último pavimento do edifício para os quatro carregamentos, obtendo-se os consumos de concreto, aço e formas, para cada um. Com estes quantitativos, partindo dos fatores de emissão de $\mathrm{CO}_{2}$ (FE) destes materiais e do seu transporte se calculou as emissões totais para cada situação, considerando a área de $352,38 \mathrm{~m}^{2}$. 
Para os cálculos das quantidades de carbono armazenadas por cada tipo de "cobertura verde", se procederam as determinações das emissões e do carbono armazenado nos substratos e na vegetação. Para o carbono dos substratos, foi feita a determinação do seu teor em cinco amostras de solos de terra orgânica usadas neste tipo de jardim. O cálculo do carbono armazenado pelo substrato é obtido por meio da multiplicação da quantidade de terra orgânica pela média do teor de carbono determinado em amostras. Para o de carbono presente nas vegetações, se utilizou estimativas unitárias por área citadas em revisões bibliográficas, multiplicadas pela área da "cobertura verde", considerando o jardim com dois anos de idade.

Multiplicando-se as quantidades de carbono por 3,667 se obteve o $\mathrm{CO}_{2}$ que foi neutralizado, procedendo-se então às comparações e verificações de viabilidade do uso das "coberturas verdes" como agentes minimizadores de emissões de $\mathrm{CO}_{2}$ para a obra estudo do caso.

\section{RESULTADOS E DISCUSSÃO}

Inicialmente foram dimensionadas as estruturas de concreto armado para suportar as quatro alternativas diferentes de "coberturas verdes", na sequencia foi calculado o total de $\mathrm{CO}_{2}$ emitido por cada uma delas, sendo então calculado o carbono armazenado em cada uma.

\subsection{Resultados do dimensionamento estrutural}

Cabe salientar que como este trabalho tem a finalidade de se fazer uma análise comparativa entre as alternativas com e sem a cobertura verde, não foi desenvolvido o projeto inteiro da estrutura do edifício, mas somente as partes da estrutura afetadas pelas cargas decorrentes da "cobertura verde". Foram estudadas a laje de cobertura e os pilares até o térreo do edifício. Portanto partes da estrutura como os demais pavimentos tipo, caixa d'água, entre outras, não foram estudadas.

Os projetos estruturais das quatro diferentes coberturas utilizaram para dimensionamento as cargas de $250 \mathrm{~kg} / \mathrm{m}^{2}$ para a impermeabilização, $50 \mathrm{~kg} / \mathrm{m}^{2}$ para carga acidental e para as alternativas de coberturas verdes CV1, CV2 e CV3, devido as diferentes alturas, as cargas de $232,5 \mathrm{~kg} / \mathrm{m}^{2} ; 387,5 \mathrm{~kg} / \mathrm{m}^{2}$ e $620,0 \mathrm{~kg} / \mathrm{m}^{2}$ 
respectivamente. Além destas cargas também foi considerado o peso próprio das diferentes estruturas.

As análises estruturais seguiram a NBR 6.118:2014, a resistência do concreto foi escolhida com o $f_{c k}$ de $25 \mathrm{MPa}$, abrangendo as quatro alternativas de carregamento, obtendo-se o dimensionando do pavimento de cobertura para estas. Os pilares de todo o edifício foram calculados para as quatro alternativas, mas as seções de concreto e das armaduras, que foram dimensionadas para o caso menos carregado (isto é sem "cobertura verde"), não necessitaram ser aumentadas para as demais situações. As áreas mínimas de armaduras e de concreto, definidas em por Norma, dos pilares já tinham capacidade de carga para todas as hipóteses. Atendendo a estas considerações, utilizando o software para cálculo estrutural de concreto armado TQS versão 18, chegou-se aos quantitativos de aço, concreto e áreas de formas de madeira.

\subsection{Resultados das emissões de $\mathrm{CO}_{2}$ pela construção das estruturas}

O cálculo das emissões geradas pelas diferentes alternativas de estruturas foi desenvolvido, procurando se aproximar da metodologia de uma ACV do "berço ao portão" com finalidade comparativa. As emissões foram calculadas a partir dos quantitativos de materiais e utilizando os Fatores de Emissão $(F E)$ para a produção e decomposição da madeira usada nas formas, para a produção do aço e do concreto. Da mesma forma se procedeu para simular o transporte destes materiais até a obra e dentro desta.

Os FE utilizados foram retirados de bibliografias disponíveis, principalmente ACV, Declarações de Impacto ambiental de produtos (EDP ou Environmental Product Declaration), que são documentos auditados. Outra fonte são levantamentos de emissões divulgados em estudos científicos. Preferencialmente foram utilizadas publicações brasileiras, mas na indisponibilidade destas utilizou-se material internacional.

Para o FE do aço considerou-se uma média de valores publicados. A EDP para barras de reforço para concreto armado, desenvolvida para os EUA, calculou FE de $0,850 \mathrm{tCO}_{2} \mathrm{eq} / \mathrm{t}$ de aço, (CMC, 2015). Outra EPD de uma siderúrgica da Turquia, que usa forno a arco elétrico, calculou $\mathrm{FE}$ de 0,802 $\mathrm{tCO}_{2} \mathrm{eq} / \mathrm{t}$ de aço, (PE 
INTERNATIONAL, 2013). A incorporadora Even em seu relatório de emissões de 2014 utilizou o FE para barras de aço de $0,619 \mathrm{tCO}_{2} \mathrm{eq} / \mathrm{t}$, citando que esta informação veio do seu fornecedor (EVEN, 2015). Com estas informações, decidiu-se para este estudo de caso utilizar para aço de concreto armado, a média destes valores, que é o FE de $0,643 \mathrm{tCO}_{2} \mathrm{eq} / \mathrm{t}$. Os resultados calculados para as emissões pelo aço utilizado estão apresentados na Tabela 03.

Para as emissões do concreto foi considerado o FE de concreto usinado. $\mathrm{O}$ FE do concreto é influenciado pela sua resistência, pelo seu consumo e tipo de cimento. Outro item significativo de emissões é o transporte entre os locais de produção dos agregados e o cimento até a usina produtora de concreto. Um detalhe específico do concreto é que parte de seu processo produtivo ocorre durante o transporte de entrega da usina até a obra, que é a mistura no caminhão betoneira, situação que é considerada neste trabalho. O estudo desenvolvido por Costa (2012) estimou as emissões relativas à produção de diversos concretos. O resultado do FE para concretos com o $f_{c k}$ de $25 \mathrm{MPa}$ foi calculado usando cimento CP II-E 32 foi de $0,366 \mathrm{tCO}_{2} / \mathrm{m}^{3}$ e com CP II-F 32 de $0,3770 \mathrm{tCO}_{2} / \mathrm{m}^{3}$. Para este estudo de caso foi escolhido utilizar a média destes valores, o $\mathrm{FE}$ de $0,3715 \mathrm{tCO}_{2} / \mathrm{m}^{3}$ de concreto.

A madeira utilizada gera emissões durante a sua produção e depois ao final da sua vida útil. A produção da madeira emite GEE em suas fases de operações florestais e industriais, sempre considerando a exploração florestal sustentável e a madeira de origem certificada. Para as emissões da produção dos materiais de madeira, foram utilizados FE para madeira serrada e FE para painéis de madeira compensada. Uso da madeira em elementos de formas e escoramento causa emissões devido a sua degradação ao final da sua vida útil, seja por queima ou degradação aeróbia. Das duas formas o carbono contido nos tecidos vegetais combina-se com o oxigênio da atmosfera formando $\mathrm{CO}_{2}$.

Para madeira serrada, considerando as emissões na floresta e na serraria, contabilizando as operações de derrubada das árvores, a transformação em toras, o transporte em caminhão até a serraria e, nesta última, as operações de corte, Campos (2012) em sua dissertação de mestrado para madeiras amazônicas, calculou FE para madeira serrada, sem o processo de secagem, com transporte variando de 0,0607 $\mathrm{tCO}_{2} / \mathrm{m}^{3}$ a $0,2300 \mathrm{tCO}_{2} / \mathrm{m}^{3}$, com mediana de $0,1118 \mathrm{tCO}_{2} / \mathrm{m}^{3}$, valor que foi escolhido para este estudo. 
Para painéis compensados, estudo utilizou como FE uma média de diversas ACV e EDP internacionais (PUETTMANN et al., 2013 e ATHENA, 2012). O valor calculado para FE foi de $0,1985 \mathrm{tCO}_{2} / \mathrm{m}^{3}$ de painel compensado.

Para as formas de madeira foi considerando que estas possuem uma reutilização de 10 vezes e uma densidade média de 0,490 t/m³. Para o carbono emitido devido à decomposição utilizou-se os teores de carbono publicados em Oliveira et al. (2011), que relacionam medições da biomassa seca de madeira para as principais espécies da região de Curitiba, utilizando-se para os cálculos que $45 \%$ de sua massa é de carbono.

Estimou-se as emissões de transporte rodoviário do aço, concreto, escoramento e forma de seu local de produção até a obra do edifício, considerando-se uma distância de $200 \mathrm{~km}$. Simulando o transporte por meio de caminhões semipesados, utilizando o fator de consumo de Diesel de 0,196 L/t/km de Costa (2012), e o FE de 3,3 $\mathrm{kgCO}_{2} / \mathrm{L}$ de Diesel usado em CBCS (2014).

Para as emissões de transporte dentro da obra, da chegada até o pavimento de aplicação, considerou-se o uso de energia elétrica (motores de elevadores) para as formas, escoramento e, aço. Para o concreto considerou-se o uso de bomba a óleo Diesel. Para a energia elétrica utilizou-se o $\mathrm{FE}$ de $0,06 \mathrm{kgCO}_{2} / \mathrm{kWh}$ fornecido pelo Ministério de Ciência e Tecnologia para a média dos anos de 2011, 2012 e 2013. Tanto para o bombeamento de concreto, como para o transporte por elevador elétrico, os tempos e consumos de energia e Diesel foram levantados em obras, acompanhandose o funcionamento destes equipamentos.

Os resultados para as emissões pelo concreto estão apresentados na Tabela 1 , as emissões pelos sistemas de formas estão na Tabela 2.

Tabela 1 - Emissões pelo concreto e aço

\begin{tabular}{|c|c|c|c|c|c|c|c|}
\hline \multirow{3}{*}{ Alternat. } & \multirow{3}{*}{$\begin{array}{c}\text { Concreto } \\
\left(\mathrm{m}^{3}\right)\end{array}$} & \multirow{3}{*}{$\begin{array}{l}\text { Aço } \\
(t)\end{array}$} & \multicolumn{5}{|c|}{ Emissões } \\
\hline & & & \multicolumn{2}{|c|}{ Concreto } & \multicolumn{2}{|c|}{ Aço } & \multirow[b]{2}{*}{$\begin{array}{l}\text { Total } \\
\left(\mathrm{tCO}_{2}\right)\end{array}$} \\
\hline & & & $\begin{array}{c}\text { Produção } \\
\left(\mathrm{tCO}_{2}\right)\end{array}$ & $\begin{array}{c}\text { Transp. } \\
\left(\mathrm{tCO}_{2)}\right.\end{array}$ & $\begin{array}{l}\text { Produção } \\
\left(\mathrm{tCO}_{2} \mathrm{eq}\right)\end{array}$ & $\begin{array}{c}\text { Transp. } \\
\left(\mathrm{tCO}_{2)}\right.\end{array}$ & \\
\hline CL & 37,1 & 1,580 & 13,783 & 2,153 & 1,016 & 0,204 & 17,156 \\
\hline CV1 & 37,3 & 1,848 & 13,856 & 2,164 & 1,188 & 0,239 & 17,449 \\
\hline CV2 & 37,3 & 2,017 & 13,856 & 2,164 & 1,297 & 0,260 & 17,579 \\
\hline CV3 & 37,8 & 2,303 & 14,043 & 2,193 & 1,481 & 0,298 & 18,015 \\
\hline
\end{tabular}

Fonte: os autores 
Tabela 2 - Emissões pelos sistemas de formas e escoramento

\begin{tabular}{c|cc|ccc|c}
\hline Alternat. & Madeira & Compensado & \multicolumn{4}{|c}{ Emissões em (tCO $\mathbf{~})$} \\
& serrada $\left(\mathbf{m}^{\mathbf{3}}\right)$ & $\left.\mathbf{1 4} \mathbf{~} \mathbf{~ m} \mathbf{( m}^{\mathbf{3}}\right)$ & Produção & Decompos. & Transp. & Total \\
\hline CL & 0,47 & 0,58 & 0,168 & 0,850 & 0,067 & 1,084 \\
CV2 & 0,47 & 0,58 & 0,170 & 0,858 & 0,067 & 1,095 \\
CV1 & 0,47 & 0,58 & 0,170 & 0,858 & 0,067 & 1,095 \\
CV3 & 0,48 & 0,60 & 0,173 & 0,874 & 0,068 & 1,115 \\
\hline
\end{tabular}

Fonte: os autores

\subsection{Resultados do carbono armazenado pelas "coberturas verdes"}

Para determinar as quantidades de carbono armazenadas no substrato, em primeiro lugar este estudo analisou cinco amostras de solo orgânico (terra preta) usual no Brasil, para estes tipos de jardins. A média dos teores de carbono, obtidos com o emprego de um analisador LECO C-144, foi de 30,24\%.

A média das densidades das amostras dos mesmos solos, medidas através do método do anel volumétrico (WHITTINGHILL, 2014) foi de $1,01 \mathrm{~g} / \mathrm{cm}^{3}$. Este valor é conservador, tendo em vista que com o tempo e o aumento da profundidade a densidade do solo tende a aumentar.

Para calcular a massa de solo utilizado por $\mathrm{m}^{2}$ em cada alternativa de cobertura, consideraram-se as espessuras de $15 \mathrm{~cm}$ para a CV1, $25 \mathrm{~cm}$ para a CV2 e $40 \mathrm{~cm}$ para a CV3, para os quais ainda foi prevista uma camada de drenagem de pedrisco de seixos com $5 \mathrm{~cm}$ de espessura. Portanto os substratos em terra orgânica ficaram em 10, 20 e $35 \mathrm{~cm}$ respectivamente.

Getter et al. (2009) em seu trabalho para "coberturas verdes" extensivas, quantificaram o carbono armazenado acima do solo com média de 0,275 kg.C/m². Lal e Augustin (2012), em seu livro citam que jardins urbanos, com cuidado intensivo, somente acima do solo seqüestram de 0,30 a $0,40 \mathrm{~kg}$ de carbono por $\mathrm{m}^{2}$ por ano.

As massas de carbono fixadas diretamente na estrutura celular da vegetação são relativamente pequenas, devido ao porte da vegetação e que apenas uma fração da sua massa ser de carbono, a maior parte da massa é composta de água.

Este trabalho considerou, para as diferentes "coberturas verdes", os valores de massa de carbono armazenada por meio do sequestro e armazenamento de $\mathrm{CO}_{2}$ do ar pelo crescimento da vegetação em dois anos, de 0,275 kg.C/m² para o CV1, de $0,600 \mathrm{~kg} . \mathrm{C} / \mathrm{m}^{2}$ para o CV2 e $0,800 \mathrm{~kg} . \mathrm{C} / \mathrm{m}^{2}$ para o CV3. Os totais de massa de carbono armazenados, e os correspondentes $\mathrm{CO}_{2}$ não emitidos, pela vegetação o solo orgânico 
do solo nas três alternativas, estão apresentados na Tabela 3. O carbono dos sistemas de raízes está contabilizado nos substratos, portanto os valores da vegetação referemse somente a parte aérea desta.

Tabela 3 - Armazenamento de carbono pelo solo orgânico e a vegetação

\begin{tabular}{cccccccc}
\hline & \multicolumn{3}{c}{ Solo Orgânico } & \multicolumn{2}{c}{ Vegetação } & \multicolumn{2}{c}{ Total armazenado } \\
\hline Alt. & $\begin{array}{c}\text { Altura } \\
(\mathbf{c m})\end{array}$ & $\begin{array}{c}\text { Massa } \\
(\mathbf{t})\end{array}$ & Carbono $(\mathbf{t})$ & $\begin{array}{c}\text { Carbono por } \\
\text { área } \\
\left(\mathbf{k g . C} \cdot \mathbf{m}^{2}\right)\end{array}$ & $\begin{array}{c}\text { Carbono } \\
(\mathbf{t})\end{array}$ & $\begin{array}{c}\text { Carbono } \\
(\mathbf{t})\end{array}$ & $\begin{array}{c}\mathbf{C O}_{2} \\
(\mathbf{t})\end{array}$ \\
$\mathbf{C V 1}$ & 10 & 35,590 & 10,764 & 0,275 & 0,097 & 10,861 & 39,827 \\
CV2 & 20 & 71,181 & 21,528 & 0,600 & 0,211 & 21,740 & 79,719 \\
CV3 & 35 & 124,566 & 37,674 & 0,800 & 0,282 & 37,956 & 139,186 \\
\hline
\end{tabular}

Fonte: os autores

Estimou-se as emissões da obtenção do substrato, composto pelo solo e pedrisco de seixo (densidade de $1,4 \mathrm{t} / \mathrm{m}^{3}$ ), baseando-se nos FE utilizados na ACV CBCS (2014), o FE para o pedrisco de seixo é $1,14 \mathrm{kgCO} / 2 \mathrm{~m}^{3}$ e o $\mathrm{FE}$ para extração de solo orgânico $0,909 \mathrm{kgCO} / \mathrm{m}^{3}$. Salienta-se que estes materiais praticamente não sofrem processos de industrialização, é quase somente a energia de extração por máquinas a Diesel. Os volumes de solo foram de 35,24; 70,48 e 123,33 $\mathrm{m}^{3}$ para as alternativas CV1, CV2 e CV3 respectivamente e o volume de pedrisco de seixo de $17,62 \mathrm{~m}^{3}$ igual para as três alternativas. As emissões calculadas para a obtenção do substrato de solo orgânico e pedrisco de seixos foram calculadas em 0,052; 0,084 e $0,132 \mathrm{tCO}_{2}$ para as alternativas $\mathrm{CV} 1, \mathrm{CV} 2$ e CV3 respectivamente.

Estimaram-se as emissões de transporte do solo e do material drenante do seu local de extração até a obra, considerando-se a distância de $25 \mathrm{~km}$. Utilizando o mesmo método e critério aplicado aos materiais da estrutura, transporte rodoviário por caminhões semi-pesados e transporte vertical dentro da obra com elevador elétrico. As emissões calculadas para o transporte do substrato de solo orgânico e pedrisco de seixos foram calculadas em 0,$976 ; 1,553$ e 2,418 $\mathrm{tCO}_{2}$ para o total destes materiais usados nas alternativas CV1, CV2 e CV3 respectivamente.

Para todo o material do substrato, somando-se o carbono armazenado e subtraindo as emissões de obtenção e transporte, calcularam-se os valores da Tabela 4 , onde estão apresentados os totais de $\mathrm{CO}_{2}$ equivalentes ao carbono armazenado e os totais por $\mathrm{m}^{2}$ de área de cobertura verde. 
Tabela 4 - Saldo do armazenamento de carbono das alternativas de coberturas

\begin{tabular}{|c|c|c|c|c|}
\hline \multirow{2}{*}{ Alternativas } & \multirow{2}{*}{$\begin{array}{c}\text { Emissões } \\
\left(\mathrm{tCO}_{2}\right)\end{array}$} & \multirow{2}{*}{$\begin{array}{l}\text { Armazenamento } \\
\left(\mathrm{tCO}_{2}\right)\end{array}$} & \multicolumn{2}{|c|}{ Saldo } \\
\hline & & & $\left(\mathrm{tCO}_{2}\right)$ & $\left(\mathrm{tCO}_{2} / \mathrm{m}^{2}\right)$ \\
\hline CV1 & 1,028 & 39,827 & 38,799 & 0,110 \\
\hline CV2 & 1,637 & 79,719 & 78,082 & 0,222 \\
\hline CV3 & 2,550 & 139,186 & 136,636 & 0,388 \\
\hline
\end{tabular}

Fonte: os autores

\subsection{Balanços das emissões e do armazenamento de carbono}

A Tabela 5 apresenta os totais de $\mathrm{CO}_{2}$ emitidos e armazenados. Nesta tabela já é possível verificar os acréscimos de emissões necessários para as alternativas estudadas. Observa-se que são positivos os saldos das emissões devido ao aumento da capacidade da estrutura quando se considera o carbono armazenado nas alternativas de coberturas verdes.

Tabela 5 - Carbono armazenado e acréscimos de emissões

\begin{tabular}{|c|c|c|c|c|c|}
\hline \multirow[b]{2}{*}{ Alternativas } & \multicolumn{2}{|c|}{ Emissões } & \multirow{2}{*}{$\begin{array}{c}\text { Armazenamento } \\
\qquad\left(\mathrm{tCO}_{2}\right)\end{array}$} & \multicolumn{2}{|c|}{ Saldo } \\
\hline & $\begin{array}{l}\text { Por alternativa } \\
\qquad\left(\mathrm{tCO}_{2}\right)\end{array}$ & $\begin{array}{c}\text { Acréscimos } \\
\left(\mathrm{tCO}_{2}\right)\end{array}$ & & $\left(\mathrm{tCO}_{2}\right)$ & $\left(\mathrm{tCO}_{2} / \mathrm{m}^{2}\right)$ \\
\hline CL & 18,240 & - & - & - & - \\
\hline CV1 & 18,543 & 0,304 & 38,799 & 38,496 & 0,109 \\
\hline CV2 & 18,674 & 0,434 & 78,082 & 77,648 & 0,220 \\
\hline CV3 & 19,130 & 0,890 & 136,636 & 135,745 & 0,385 \\
\hline
\end{tabular}

Fonte: os autores

\subsection{Discussão}

Analisando as "coberturas verdes", o primeiro ponto a se ressaltar é a sua enorme capacidade de armazenar carbono. Neste estudo de caso estas coberturas apresentaram um saldo favorável de armazenamento de carbono de $0,109 \mathrm{tCO} / \mathrm{m}^{2}$ para a $\mathrm{CV} 1,0,220 \mathrm{tCO} / 2 \mathrm{~m}^{2}$ para a $\mathrm{CV} 2$ e $0,385 \mathrm{tCO}_{2} / \mathrm{m}^{2}$ para a CV3. Estes resultados condizem com os valores obtidos por Whittinghill et al. (2014), no qual foram medidas quantidades de carbono armazenados de até $0,239 \mathrm{tCO}_{2} / \mathrm{m}^{2}$ (ou $0,0652 \mathrm{tC} / \mathrm{m}^{2}$ ). O trabalho Whittinghill et al. (2014) analisou "coberturas verdes" com três anos de idade com um substrato de $10,5 \mathrm{~cm}$ de espessura. Nos paises desenvolvidos de clima frio, é comum o uso de substratos de misturas de areia, silte e argila e fertilizantes, que inicialmente contém menor quantidade de carbono armazenado, mas absorvem mais com o tempo (WHITTINGHILL et al., 2014). 
Os resultados provam que as quantidades de carbono armazenadas, para 0 estudo de caso, são muito maiores que as emissões decorrentes do reforço necessário das estruturas para que estas suportem as "coberturas verdes". Embora obviamente estes resultados sejam específicos para o edifício estudado, o saldo muito favorável de armazenamento de carbono, quase com certeza apresenta as coberturas verdes como uma forma significativa de aproximar a construção dos edifícios da meta de serem "neutras em carbono".

Mesmo com um eventual uso de FE maiores para o cimento e o aço (tipos de cimento com FE maiores e siderúrgicas com maiores emissões), a superioridade dos valores de armazenamento de carbono deve permanecer.

Portanto a implantação das "coberturas verdes" é extremamente positiva no contexto da neutralização das emissões de GEE pelas obras, além de favorecer o desempenho térmico das edificações, característica que minimiza também as emissões durante a etapa de uso da edificação.

Pode-se avaliar o impacto de neutralização de emissões causado pelas "coberturas verdes", verificando o quanto sua implantação afeta uma obra completa. Partindo das informações das obras de uma grande construtora brasileira que publica anualmente seus relatórios de emissões de GEE para seus edifícios de apartamentos, construídos em concreto armado, que apresenta uma média de emissões de 167,1 $\mathrm{kgCO}_{2} / \mathrm{m}^{2}$ de área construída (EVEN, 2015), observa-se que as alternativas CV1, CV2 e CV3 neutralizam respectivamente o equivalente a 65\%, 132\% e 230\% de sua área construída. Portanto uma "cobertura verde" intensiva (CV3) pode neutralizar as emissões de construção de mais de dois pavimentos do edifício. Cabe ressaltar que nos relatórios de emissões das obras estão englobadas inclusive as emissões da remoção da vegetação original do terreno.

Como comparativo deste fator de emissão calculado para alguns edifícios Brasileiros, podemos utilizar os resultados das ACV desenvolvidas para edifícios residenciais na Espanha e na Alemanha, publicadas pelo ENSLIC Building, que é um projeto dentro da comissão da União Europeia. Estas ACV apresentaram fatores de emissão de 193,0 a 404,0 kgCO $/ \mathrm{m}^{2}$ (ENSLIC BUILDING, 2010a e ENSLIC BUILDING, 2010b). Esclarece-se aqui que em geral as ACV produzem FE maiores que os relatórios de emissões, salienta-se também que edifícios europeus, devido a maior 
necessidade de isolamento térmico, tendem a ter fatores de emissão superiores aos brasileiros.

\section{CONCLUSÃO}

Com os resultados apresentados pode-se concluir que:

- É muito pequeno o aumento das emissões de $\mathrm{CO}_{2}$ necessário para que uma estrutura possa suportar o acréscimo de carga de uma "cobertura verde";

- É grande a eficácia das "coberturas verdes" no objetivo de fixar carbono e neutralizar parte das emissões decorrentes da implantação das obras de edifícios;

- Este estudo considerou a vegetação da "cobertura verde" com a idade de dois anos, embora com o passar do tempo a quantidade de carbono presente na vegetação ainda seja crescente por um período, até a estabilização do jardim;

- Os substratos armazenam muito mais carbono que a parte aérea da vegetação, representando para as três alternativas do estudo de caso $99 \%$ do total de carbono armazenado.

- A implantação de "coberturas verdes" nas coberturas dos edifícios tem a forte tendência de alcançar saldos de carbono muito favoráveis, armazenando muito mais que às emissões decorrentes da sua implantação.

Diante do exposto, para a finalidade de neutralizar as emissões de $\mathrm{CO}_{2}$ de uma obra de construção, a implantação de "coberturas verdes" mostrou balanços entre armazenamento e emissões, amplamente favoráveis.

\section{AGRADECIMENTOS}

Os autores agradecem aos engs. Moacir e Fabio Inoue pelo auxílio no desenvolvimento do projeto estrutural utilizado neste trabalho, ao meu orientador Carlos Sanquetta pela ajuda conceitual geral e aos profissionais do laboratório do BIOFIX da UFPR pela execução dos ensaios laboratoriais. 


\section{REFERÊNCIAS}

ABNT. NBR 6120:1980 Versão Corrigida: 2000. Cargas para o cálculo de estruturas de edificações. ABNT - Associação Brasileira de Normas Técnicas, 30/11/1980.

ABNT. NBR 6118: 2014 Versão Corrigida: 2014. Projeto de estruturas de concreto Procedimento. ABNT - Associação Brasileira de Normas Técnicas, 29/04/2014.

ABNT. NBR ISO 14025: 2015. Rótulos e declarações ambientais - Declarações ambientais de Tipo III - Princípios e procedimentos . ABNT - Associação Brasileira de Normas Técnicas, 23/01/2015.

ABNT. NBR ISO 14040:2009 versão corrigida 2014 e Gestão ambiental - Avaliação do ciclo de vida - Princípios e estrutura. ABNT - Associação Brasileira de Normas Técnicas, 21/07/2014.

ABNT. NBR ISO 14044: 2009 Errata 1:2014. Gestão ambiental - Avaliação do ciclo de vida Requisitos e orientações. ABNT - Associação Brasileira de Normas Técnicas, 21/07/2014.

ABNT. NBR ISO 14064: 2007 Gases de efeito estufa. ABNT - Associação Brasileira de Normas Técnicas, 11/05/2007.

ATHENA. A Cradle-to-Gate Life Cycle Assessment of Canadian Softwood Plywood Sheathing- an Update. Athena Sustainable Materials Institute - Prepared for: The CertiWood ${ }^{\mathrm{TM}}$ Technical Centre, October 2012. Disponível em:

<http://www.canply.org/english/news/dec2012/CDN\%20Plywood\%20LCA\%20Final\%20Report\%2 0Oct\%2028 2012.pdf> Acesso em 10 jun. 2016.

CBCS. Projeto avaliação de ciclo de vida modular de blocos e pisos de concreto. CBCS Conselho Brasileiro de Construção Sustentável. Novembro de 2014. Disponível em:<http://www.cbcs.org.br/ 5dotSystem/userFiles/ACV-m/CBCS ACV-blocos-concreto.pdf >

CAMPOS, E.F. Emissão de $\mathbf{C O}_{2}$ da madeira serrada da Amazônia: o caso da exploração convencional. Escola Politécnica, São Paulo, dissertação de mestrado, 2012. Disponível em: $<$ http://www.teses.usp.br/teses/disponiveis/3/3146/tde-12062013-170523/pt-br.php> Acesso em 10 jun. 2016.

CASTLETON, H.F.; STOVIN, V.; BECK, S. B. M.; DAVIDSON, J.B. Green roofs; building energy savings and the potential for retrofit. Elsevier, Energy and Buildings, 42, p. 1582-1591, 2010. Disponível em: <http://www.e-

futures.group.shef.ac.uk/publications/pdf/41 Green\%20Roofs\%20Castleton.pdf > Acesso em 10 jun. 2016.

CMC. EPD - Concrete Reinforcing Steel. Commercial Metals Company (CMC), Environmental Product Declaration of Concrete Reinforcing Steel, EPD-012, 2015.09.15. Disponível em: $<$ http://www.astm.org/CERTIFICATION/DOCS/216.EPD for Concrete Reinforcing Steel.pdf> Acesso em 10 jun. 2016.

COSTA, B. L. C. Quantificação das emissões de $\mathbf{C O}_{2}$ geradas na produção de materiais utilizados na construção civil. Rio de Janeiro, 2012, Dissertação (Mestrado) -Pós-graduação em Engenharia Civil, COPPE - UFRJ. Disponível em: $<$ http://www.coc.ufrj.br/index.php/component/docman/doc download/1215-bruno-luis-decarvalho-da-costa-mestrado? Itemid=> Acesso em 10 jun. 2016. 
ENSLIC BUILDING. D 4.2. CIRCE. Pilot Case 3. ENSLIC - Energy Saving through Promotion of Life Cycle Assessment in Buildings, 2010a. Disponível em

$<$ https://ec.europa.eu/energy/intelligent/projects/sites/iee-

projects/files/projects/documents/enslic building lca case studies in spain en.pdf> Acesso em 10 jun. 2016.

ENSLIC BUILDING. LCA of Housing Building in Frankfurt - Case 18. ENSLIC - Energy Saving through Promotion of Life Cycle Assessment in Buildings, 2010b. Disponível em:

$<$ http://circe.cps.unizar.es/enslic/texto/cs 18.pdf> Acesso em 10 jun. 2016.

EVEN. RELATÓRIO DE EMISSÕES DE GASES DE EFEITO ESTUFA - Inventário 2014. EVEN Construtora e Incorporadora S/A, setembro de 2015. Disponível em:

<http://www.even.com.br/sustentavel/wp-content/uploads/2015/09/Even RELATORIOEMISSOES-2015.pdf> Acesso em 10 jun. 2016.

GARRISON, N.; HOROWITZ, C. Looking Up: How Green Roofs and Cool Roofs Can Reduce Energy Use, Address Climate Change, and Protect Water Resources in Southern California. Natural Resources Defense Council, EMMET CENTER ON CLIMATE CHANGE AND THE ENVIRONMENT, UCLA SCOOL OF LAW. june 2012. Disponível em:

<http://www.nrdc.org/water/pollution/files/greenroofsreport.pdf> Acesso em 10 jun. 2016.

GETTER, K. L.; ROWE D. B.; ROBERTSON, G. P.; CREGG, B. M. and ANDRESEN, J. A. Carbon Sequestration Potential of Extensive Green Roofs. Environment. Science and Technology, v. 43, n. 19, p. 7564-7570, 2009. Disponível

em:<http://lter.kbs.msu.edu/docs/robertson/Getter2009EnvSciTech.pdf > Acesso em 10 jun. 2016.

JOHNSON, J. and NEWTON, J. BUILDING GREEN. A Guide to using on Roofs, Walls and Pavements. Staffordshire University, The London Ecology Unit, London, 1991.

LAL, R. and AUGUSTIN, B. Carbon Sequestration in Urban Ecosystems. Springer, 2012.

LI, W.C.; YEUNG, K.K.A. A comprehensive study of green roof performance from environmental perspective. Elsevier, International Journal of Sustainable Built Environment, v. 3, n. 1, June p. 127-134, 2014. Disponível em: <http://ac.els-cdn.com/S2212609014000211/1-s2.0-

S2212609014000211-main.pdf? tid=4a8da4e4-ca6f-11e5-836a-

00000aacb35d\&acdnat $=1454501684$ 28d2017a77b590685198a080930b5d61 > Acesso em 10 jun. 2016.

OLIVEIRA, E. O.; NAKAJIMA, N. Y.; CHANG, M.; HALISKI, M.; Determinação da quantidade de madeira carbono e renda da plantação florestal. EMBRAPA FLORESTAS, Colombo PR, 2011. Disponível em:

<http://www.infoteca.cnptia.embrapa.br/bitstream/doc/898993/1/Doc220.pdf > Acesso em 10 jun. 2016.

PE INTERNATIONAL. Reinforcing Steel - DILER DEMIR ÇELIKK. PE INTERNATIONAL AG. Leinfelden-Echterdingen, Alemanha November 2013. Disponível em:

<http://www.dilerhld.com/upload/EPD Report Diler Reinforcing 11Nov2013rev3.pdf>.

PUETTMANN, M.; ONEIL E.; WILSON, J.; JOHNSON, L. Cradle to Gate Life Cycle Assessment of Softwood Plywood Production from the Pacific Northwest. Updated april 2013. Disponível em: 
<http://www.corrim.org/pubs/reports/2013/phase1 updates/PNW\%20Plywood\%20LCA\%20final\% 20May\%202013.pdf> Acesso em 10 jun. 2016.

ROWE, D.B. Green roofs as a means of pollution abatement. Elsevier, Environmental Pollution, 2010. Disponível em: <http://www.ncbi.nlm.nih.gov/pubmed/21074914> Acesso em 10 jun. 2016.

SANTAMOURIS, M. Cooling the cities - A review of reflective and green roof mitigation technologies to fight heat island and improve comfort in urban environments. Solar Energy, 103 (2014) 682-703. Disponível em: <http://www.coolrooftoolkit.org/wp-

content/uploads/2015/03/Cooling-the-cities-\%E2\%80\%93-A-review-of-reflective-and-green-roofmitigation-technologies-to-fight-heat-island-and-improve-comfort-in-urban-environments.pdf> Acesso em 10 jun. 2016.

UNEP. Buildings and Climate Change - Summary for Decision-Makers. United nations environment Programme - Sustainable Buildings \& Climate Initiative. UNEP DTIE - Sustainable Consumption \& Production Branch, Paris, France, 2009.

WARK, C. G. and WARK, W. W. Green Roof Specifications and Standards, Establishing an emerging technology. The Construction Specifier, v.56, n. 8, August 2003. Disponível em:<http://www.greenroofs.com/pdfs/newslinks-803 construction specifier.pdf> Acesso em 10 jun. 2016.

WHITTINGHILL, L. J.; D. ROWE, D. B.; SCHUTZKI, R.; CREGG, B. M. Quantifying carbon sequestration of various green roof and ornamental landscape systems. Landscape and Urban Planning, v.123, March 2014, Pages 41-48. Disponível em:

<http://www.sciencedirect.com/science/article/pii/S0169204613002296> Acesso em 10 jun. 2016. 